\title{
Streaming Instabilities in Converging Geometry
}

\author{
Lucas I. Inigo Gamiz ${ }^{1}$, Bernhard Ersfeld ${ }^{1}$, Samuel R. Yoffe ${ }^{1}$, R. Alan Cairns ${ }^{2}$, and Dino A. \\ Jaroszynski $^{1}$ \\ ${ }^{1}$ Department of Physics, SUPA, University of Strathclyde, Glasgow G4 0NG United Kingdom \\ ${ }^{2}$ School of Mathematics and Statistics, University of St Andrews, St Andrews KY16 9SS, \\ United Kingdom
}

\begin{abstract}
We present an investigation into counter-streaming electron beams converging towards, and diverging from, a single point in two dimensions, leading to two-stream and current filamentation instabilities, which have radial and azimuthal density modulations, respectively. Using a semi-analytical approach and numerical simulations, we find no evidence for the two-stream instability in this geometry, but show that the system is unstable to the development of current filamentation.
\end{abstract}

Keywords: Two-stream instability, current filamentation instability, plasma instabilities, streaming instabilities

\section{INTRODUCTION}

Fluid flow can lead to several types of interactions in multiple fluid flow, which can result in instabilities. These occur in a wide range of fluids that are common in nature, for example the Kelvin-Helmholtz instability observed in cloud formation. Instabilities occur in fluids with a velocity sheer, where two fluids with different velocities flowing relative to each other or at boundaries between fluids. The discontinuity in velocity causes the fluid to "curl" into the opposite population causing waves to be formed. This is observed in wind flowing above water, clouds, and even in the sun's corona. ${ }^{1}$ As instabilities progress, the energy of the fluids is transferred to turbulence, which can lead to an overall disruption of the flow. Plasma is also subject to instabilities ${ }^{2-4}$ driven by, e.g., anisotropic temperatures ${ }^{5}$ counter-propagating particle flows, ${ }^{6,7}$ or inhomogeneous densities. ${ }^{7}$ These include two-stream and current filamentation, Kelvin-Helmholtz and Rayleigh-Taylor instabilities. Plasmas are central to achieving nuclear fusion in magnetic (tokamaks) ${ }^{3}$ or inertial confinement, acceleration of charged particles using wakefields, ${ }^{8}$ and astrophysical phenomena such as gamma ray bursts from galaxies ${ }^{2}$ and first-order Fermi acceleration. ${ }^{2}$ The study presented here focuses on two-stream and current filamentation instabilities in converging geometries, which should have wide application.

\section{TWO-STREAM AND CURRENT FILAMENTATION INSTABILITIES}

Plasma instabilities can arise from particle populations flowing relative to each other where a classic example is the two-stream instability (TSI). ${ }^{3,7}$ Modes develop with wavevectors parallel to the direction of propagation, making this instability electrostatic in nature. When particle populations overlap, their mutual perturbation leads to out-of-phase oscillations and bunching of particle populations and corresponding electric fields. Using a fluid model we linearise the continuity and momentum equations, and use Gauss's Law to obtain a dispersion relation for wavenumber and frequency. We assume perturbations with a harmonic dependence $\psi(r, t)=\psi \exp [i(\boldsymbol{k} \cdot \boldsymbol{r}-\omega t)]$ where $\omega$ is the frequency and $\boldsymbol{k}$ is the wave vector of the perturbation. The growth rates are given by the positive imaginary parts of the complex solutions of the frequency. In the cold, non-relativistic and homogeneous case, the dispersion relation has the form

$$
D(\omega, k)=\omega_{p}^{2}\left(\frac{1}{(\omega-k \bar{u})^{2}}+\frac{1}{(\omega+k \bar{u})^{2}}\right)-1=0,
$$

Further author information: (Send correspondence to D.A. Jaroszynski) DAJ: E-mail: d.a.jaroszynski@strath.ac.uk and LIIG: lucas.inigo-gamiz@strath.ac.uk 
where $\bar{u}$ is the equilibrium velocity of the particles, $\omega_{p}=\sqrt{\bar{n} e^{2} /\left(m \epsilon_{0}\right)}$ the plasma frequency, $e$ is the electron charge, $\bar{n}$ is the equilibrium number density, $m$ is the mass of the particles, and $\epsilon_{0}$ the free space permittivity. There are two singularities in this dispersion relation, $\omega= \pm k u$, corresponding to the frequency shifts for the fluids streaming with, or counter to, the propagating modulations. Furthermore, the growth rate can be obtained by solving for the square of the frequency

$$
\omega^{2}=k^{2} \bar{u}^{2}+\omega_{p}^{2} \pm \omega_{p}^{2} \sqrt{1+\frac{4 k^{2} \bar{u}^{2}}{\omega_{p}^{2}}} .
$$

One is negative for $0<k \bar{u}<\sqrt{2} \omega_{p}$, which corresponds to the growth rate

$$
\Gamma_{T S I}=\sqrt{\omega_{p}^{2} \sqrt{1+\frac{4 k^{2} \bar{u}^{2}}{\omega_{p}^{2}}}-k^{2} \bar{u}^{2}-\omega_{p}^{2}} .
$$

The cutoff at $k=\sqrt{2} \omega_{p} / \bar{u}$ restricts the two-stream instability to small wavenumbers.

The current filamentation instability, on the other hand, leads to density modulations with wavevectors perpendicular to the propagation direction. These are electromagnetic in nature. As the streams of charged particles propagate through each other, they interact through magnetic repulsion of counterstreaming current filaments, while attracting co-moving particles. An imbalance due to density modulations gives rise to a net force that enhances the modulation..$^{5,6}$

In the same way as for the two-stream instability, the current filamentation instability can be studied by obtaining a dispersion relation from the linearised continuity, momentum and Maxwell's equations

$$
D(\omega, k)=\omega^{2}-c^{2} k^{2}-2 \omega_{p}^{2}-\frac{2 \omega_{p}^{2} k^{2} \bar{u}^{2}}{\omega^{2}}=0,
$$

which can be solved to obtain the unstable modes that have a growth rate

$$
\Gamma_{C F I} \approx \frac{\bar{u} k}{\sqrt{1+\frac{c^{2} k^{2}}{2 \omega_{p}^{2}}}} .
$$

This increases asymptotically to $\sqrt{2} \omega_{p} \bar{u} / c$ as $k \rightarrow \infty$. One can compare the two-stream and current filamentation growth rates and the ranges where their growth is finite. The two-stream and the current filamentation instabilities are observed in Fig 1 to have the same growth rate for small $k$. However, the current filamentation instability exhibits growth over a larger range of wavenumbers. The unstable modes grow as long as the particle populations interact and the instability reaches saturation when the modulation becomes comparable with the background density. From Fig 1, one can observe how the difference in modulation direction can change the growth rate. Note that arbitrary angles between the streaming velocity and the modulation wave vector results in hybrids of both instabilities. ${ }^{9,10}$ Due to the larger range of wave numbers, it is more common to observe the current filamentation instability in nature than the two-stream instability.

\section{SEMI-ANALYTICAL APPROACH}

Plasma streams flowing in converging geometries are found in nature and man-made "laboratories", including stellar, space plasma and inertial confinement fusion. Converging geometry refers to a flow that converges to a specific point, which leads to a number density that depends on position. The specific type of geometry to be studied in this case is a disk with two particle populations, one propagating radially inwards, whilst the other propagates radially outwards. Both populations, in this case electrons, interact when they pass through each other. In a homogeneous system, this would satisfy the criteria for streaming instabilities to occur. The dynamics of the electrons in this geometry is studied for a cold, non-relativistic plasma using the the continuity, momentum, and Maxwell's equations to obtain dispersion curves. This is a challenging task because the equilibrium number 


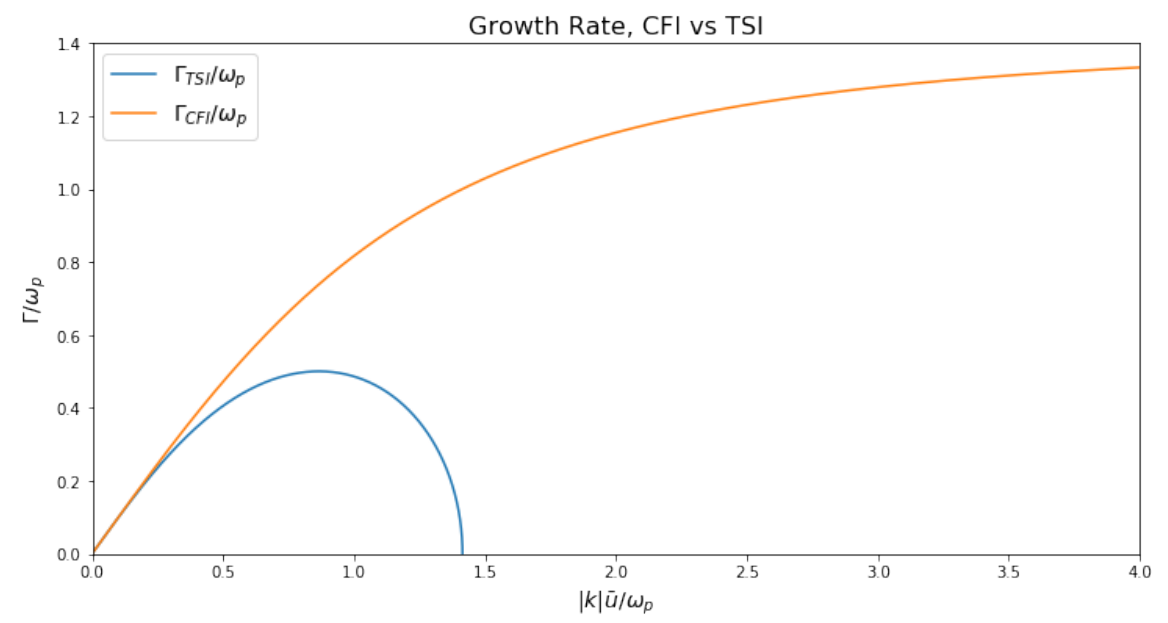

Figure 1. The growth rate for the two-stream and current filamentation instabilities, showing that they have different ranges. The two-stream instability has a cutoff at $k=\sqrt{2} \omega_{p} / \bar{u}$, while the current filamentation instability continues to exhibit growth beyond this cutoff.

density is not homogeneous. We assume a constant unperturbed radial velocity in the continuity equation and a time independent unperturbed number density when deriving the general form for the equilibrium number density, $\bar{n} \propto 1 / r$, where $r$ is the distance from the centre of the disk and the bar denotes the equilibrium solutions. Since the equilibrium number density depends on $r$, it is no longer useful to take the Fourier transform with respect to $r$. It would involve convolutions with the Fourier transforms of the background density and therefore would not lead to a simple replacement of the derivative $\partial_{r} \rightarrow i k_{r}$. However, we can still assume that the perturbations of number density $\tilde{n}$, velocity $\tilde{u}$ and electromagnetic fields $\tilde{E}_{r}, \tilde{E}_{\theta}, \tilde{B}_{z}$ have a harmonic dependence $\psi(r, \theta, t)=\psi(r) \exp [i(l \theta-\omega t)]$ on time $t$ and azimuthal angle $\theta$, allowing substitutions of the form $\partial_{\theta}=i l, \partial_{t}=-i \omega$. Local or WKB approximations can only be utilized for large $r$, where the density varies slowly. The latter assumes $\left|\partial_{r} k_{r}\right| \ll\left|k_{r}\right|^{2}$ and as $r \rightarrow 0$ the wavenumber can vary rapidly.

The radial dependence of the perturbations is determined from a set of coupled ordinary differential equations for a general case with azimuthal dependence

$$
\begin{aligned}
\partial_{r} \tilde{n}_{ \pm} & =\left[\frac{i \omega}{\bar{u}_{ \pm}}-\frac{1}{r}\right] \tilde{n}_{ \pm}-i \omega \bar{n} \tilde{u}_{ \pm, r}-\frac{i l \bar{n}}{\bar{u}_{ \pm}, r}+\frac{\bar{n}}{\bar{u}_{ \pm}^{2}} \tilde{E}_{r}, \\
\partial_{r} \tilde{u}_{ \pm, r} & =-\frac{e}{m \bar{u}_{ \pm}} \tilde{E}_{r}+\frac{i \omega}{\bar{u}} \tilde{u}_{ \pm, r}, \\
\partial_{r} \tilde{u}_{ \pm, \theta} & =-\frac{e}{m \bar{u}_{ \pm}}\left[\tilde{E}_{\theta}-\bar{u}_{ \pm} \tilde{B}_{z}\right]+\frac{i \omega}{\bar{u}_{ \pm}} \tilde{u}_{ \pm, \theta}, \\
\partial_{r} \tilde{E}_{r} & =-\left(\tilde{n}_{+}+\tilde{n}_{-}\right)-\frac{1}{r} \tilde{E}_{r}-\frac{i l}{r} \tilde{E}_{\theta}, \\
\partial_{r} \tilde{E}_{\theta} & =i \omega \tilde{B}_{z}-\frac{\tilde{E}_{\theta}}{r}+\frac{i l}{r} \tilde{E}_{r}, \\
\partial_{r} \tilde{B}_{z} & =i \omega \tilde{E}_{\theta}+\bar{n}\left[\tilde{u}_{+, \theta}+\tilde{u}_{-, \theta}\right],
\end{aligned}
$$

where the subscripts \pm denote the populations propagating radially outwards and inwards, respectively, tilde denotes perturbations, bar the equilibrium components with $\bar{u}_{+}=-\bar{u}_{-}=\bar{u}$, and both populations have the same equilibrium number density $\bar{n}_{+}=\bar{n}_{-}=\bar{n}$. These equations can be summarized as

$$
N^{\prime}-\Lambda N=0,
$$

where $N$ is a vector containing the perturbed components, $N^{\prime}$ is the derivative of vector $N$ with respect to the radial coordinate $r$, and $\Lambda$ is a matrix with the coefficients (some of which depend on $r$ ) multiplying the 
perturbations. The boundary conditions are set so that every vector is 0 at the origin, and to be continuous, $\partial_{r} \tilde{E}_{r}=0$ at $r=0$, therefore the number densities are $\tilde{n}_{+}=-\tilde{n}_{-}$. The general matrix enables solutions for the
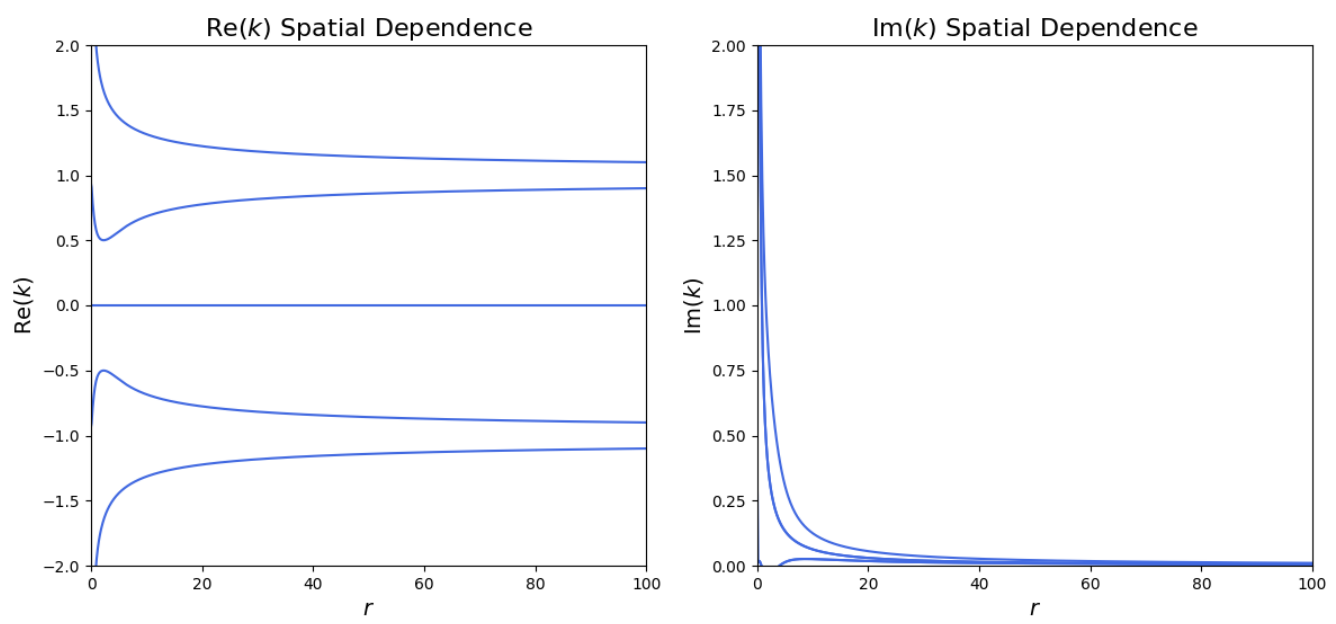

Figure 2. Real parts (left) and imaginary parts (right) of $i k$ of the reduced matrix $\Lambda$ for the two-stream case. The former correspond to inward and outward propagation of perturbations, while the latter corresponds to decay or growth of their amplitudes with $r$.

two-stream instability to be obtained by setting $l$, the azimuthal electric field and velocities, and the magnetic field to zero, which eliminates an azimuthal dependence.

The results for this case do not show evidence of an instability. The eigenvalues $\lambda=i k$ of the matrix $\Lambda$ yield radial wave numbers $k$ that depend on $r$. The real parts of the wave numbers correspond to inward and outward propagating waves for $\operatorname{Re}[k]<0$ and $\operatorname{Re}[k]>0$, respectively. The imaginary part of $k$ gives the radial dependence of the modulation amplitude; $\operatorname{Im}(k)>0$ corresponds to decreasing amplitudes as $r$ increases. Fig 2 shows no coalescence of $k$-branches corresponding to incoming and outgoing waves, which implies no instability. There is a solution that is purely decaying, but this is due to the geometric effect resulting from convergence or
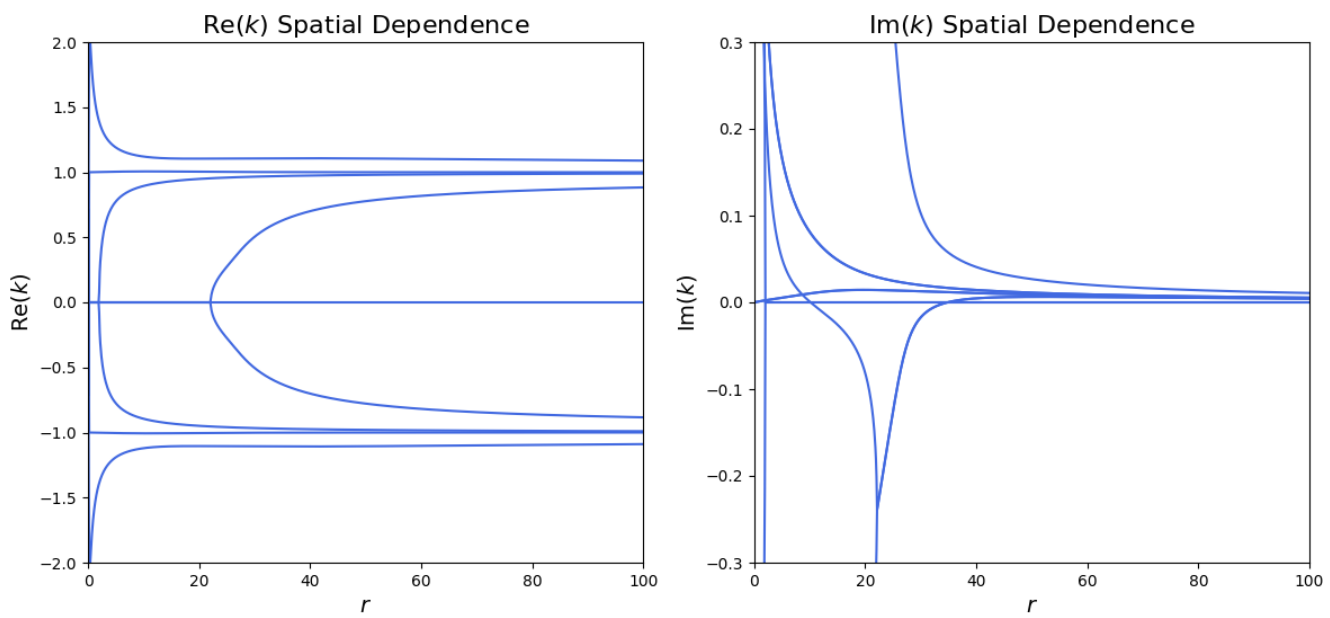

Figure 3. Real parts (left) and imaginary parts (right) of the eigenvalues $i k$ of the full matrix $\Lambda$ for the current filamentation case, with $l=23$. The former correspond to inward and outward propagation of perturbations while the latter to decay or growth of their amplitudes with $r$. 
divergence of the flow and does not indicate an instability.

As the current filamentation instability exists over a larger range of wavenumbers in homogeneous plasma, it is likely that instabilities also occur in the inhomogeneous case. The search for this kind of instability proceeds in the same way as for the two-stream case described above, but now the analysis includes the azimuthal components of the velocity perturbation and electric field, an axial magnetic field, and dependence on the azimuthal angle $\theta$.

Fig 3 demonstrates that negative and positive real parts of the complex wave number $k$ (incoming and outgoing waves) coalesce for decreasing $r$, near $r=22.1$ and $r=2.1$, respectively, while simultaneously the corresponding imaginary components of $k$ bifurcate. This provides evidence of an instability.

\section{SIMULATIONS}

The converging plasma beams are simulated using the particle-in-cell code, EPOCH. ${ }^{11}$ The initial conditions are two ring-shaped electron bunches respectively propagating radially inwards and radially outwards arranged so they can cross and interact in a similar way to that of the semi-analytical approach described above.

The two annular bunches will propagate with opposite equal velocities and because their charge density varies as the bunches propagate, they are set up to have the same charge at the time of complete overlap with a number density of $n_{ \pm}=10^{25} \mathrm{~m}^{-3}$, with a relativistic factor of $\gamma=2$. The bunches have a width of $2.2 \mu \mathrm{m}$ and are separated by $4.4 \mu \mathrm{m}$.
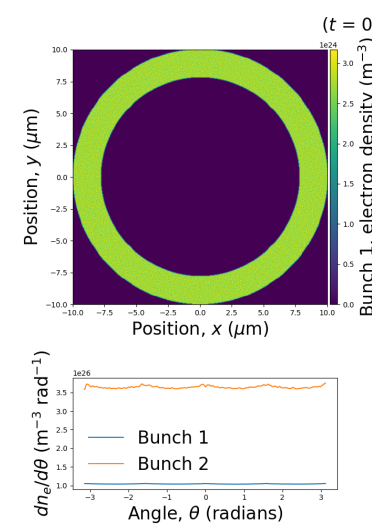

Figure 4. The initial conditions for two counterstreaming electron bunches. LHS/RHS showing inward/outward propagating bunches (bunch 1 and bunch 2) in a colour coded plot. A number density per angle of both bunches is beneath bunch 1 and a Fourier transform of the density as a function of angle is shown below bunch 2 .
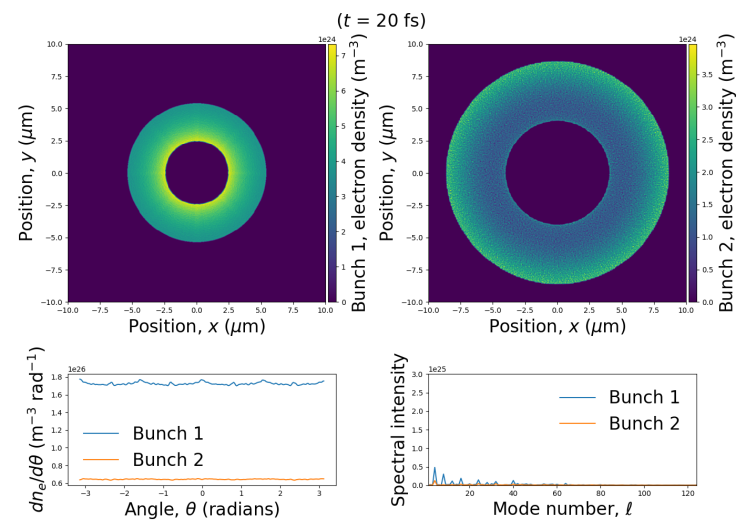

Figure 5. Plot showing the density and Fourier transform after the beams have crossed.)

One would expect filament-like structures to develop due to the current filamentation instability. However, Fig. 4 and Fig. 5 do not show obvious emergence of any structures provoked by this instability. It is reasonable to assume that the lack of growth is due to the short interaction time for instabilities to develop from noise and geometrical effects due to radial expansion. To test this, an initial modulation of one of the bunches is implemented to initiate the instability. This requires a radial modulation for the two-stream instability, i.e., parallel to the propagation of the bunches with a wavelength chosen to be in the unstable regime. This is achieved by imposing a small amplitude (10\% of the average) sinusoidal modulation in the number density. When the bunches interact, this should instigate modulation as in the initially homogeneous bunch, which should, in turn, enhance the original modulations if there is an instability. This should show as an increase in signal strength, i.e., modulation amplitude in both bunches. Furthermore, the modulations should be out of phase by a factor of $\pi$.

In Fig 7 showing the density after interaction, no modulation develops in the unmodulated bunch. This confirms the semi-analytical results, which show that two-stream type instability does not develop. 

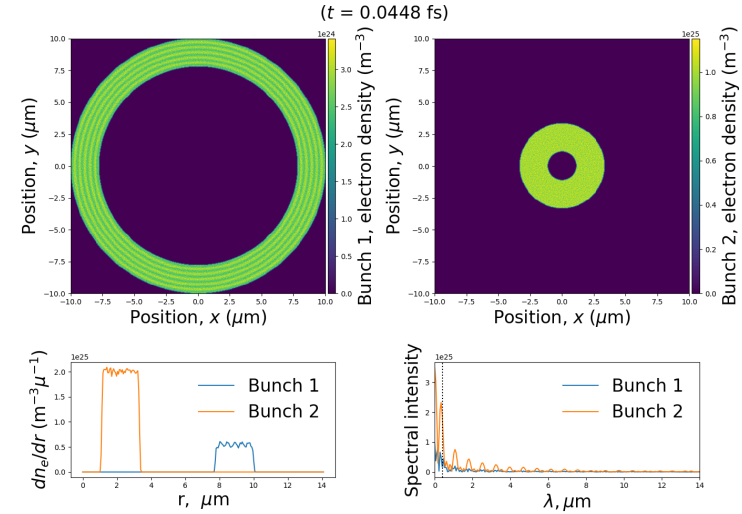

Figure 6. As in the previous figure but with a $10 \%$ sinusoidal modulation in the number density of bunch 1 whilst bunch 2 is unmodulated. Below, the azimuthal density plot for both bunches and its Fourier transform.

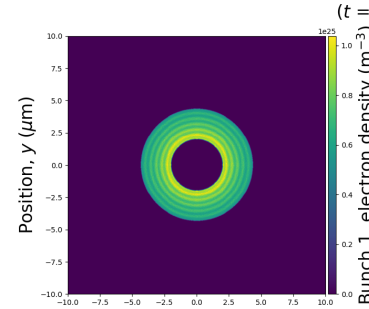

Position, $x(\mu \mathrm{m})$
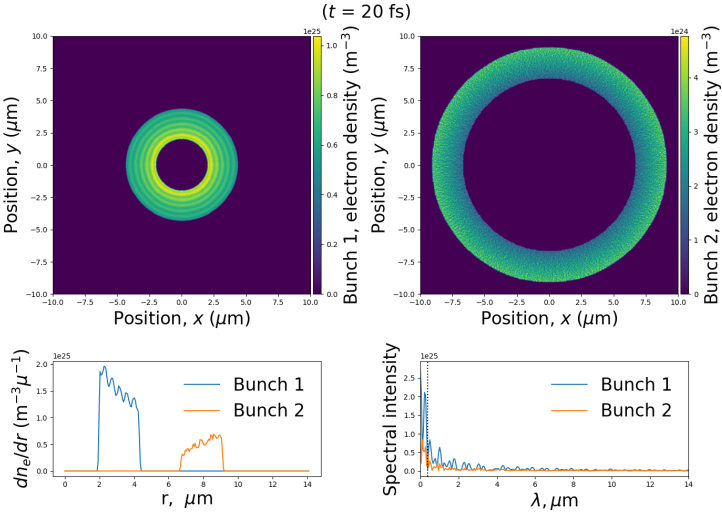

Position, $x(\mu \mathrm{m})$

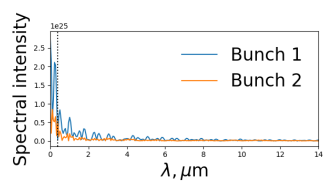

Figure 7. As in Fig. 6 but after interaction.

The current filamentation instability leads to current modulations with wavevectors perpendicular to the propagation direction. To seed such instabilities, a sinusoidal modulation in the number density of strength $10 \%$ is imposed in the azimuthal direction. Apart from this, the simulation parameters are exactly the same as in
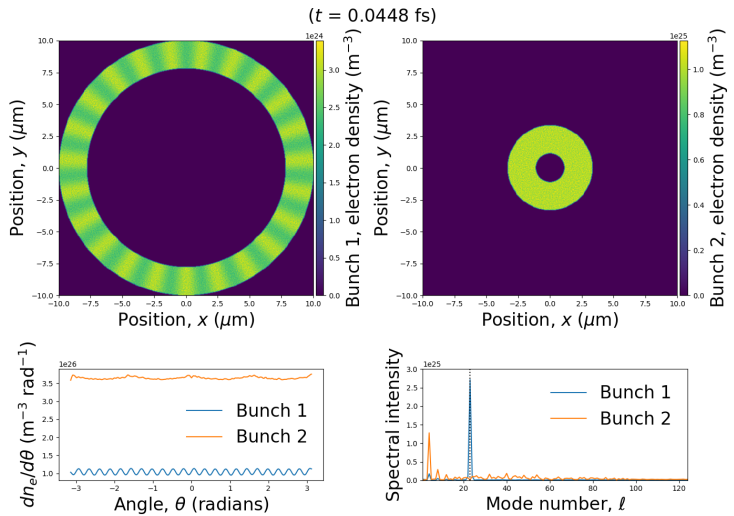

Figure 8. initial conditions with $10 \%$ of the average density modulation in the azimuthal direction (bunch 1).

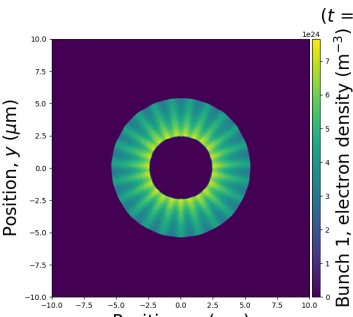

Position, $x(\mu \mathrm{m})$
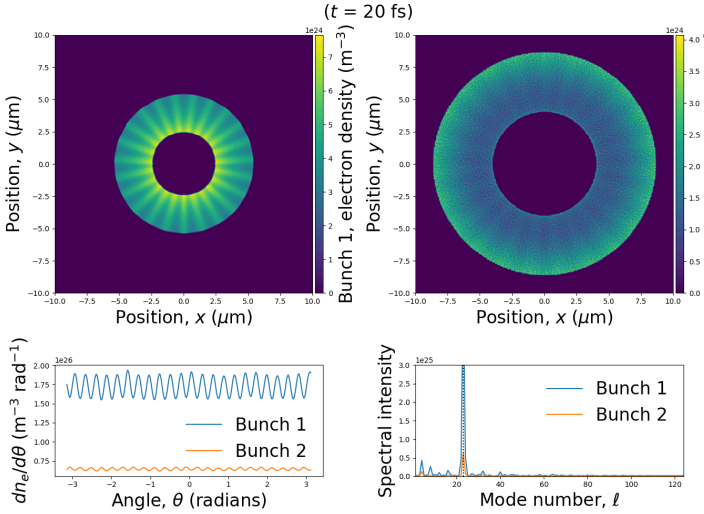

Figure 9. Timestep at 20 fs demonstrating a modulation present in bunch 1 post-interaction.

the previous case. An instability would result in modulations of both bunches out of phase by $\pi$. A modulation develops in the unmodulated bunch with a phase difference of $\approx \pi$. Fig. 8 and Fig. 9 show evidence of the current filamentation instability through an increase in signal strength of the modulation, and a phase difference of $\pi$ between the two modulations. The amplitude of the seed modulation grows before interaction with the second bunch because of the increase in density as the seeded bunch moves inwards. However, during interaction the second bunch picks up the modulation at $l=23$ even though the density of this bunch decreases, which suggests that the effect of the instability is stronger than the geometric effects of the expansion. The extra modulation at $l=4$, is an effect of the Cartesian simulation grid.

\section{CONCLUSIONS}

We have investigated instabilities when counter-streaming electron beams interact as they converge/diverge towards/away from a point in two dimensions. Semi-analytical results provide no evidence of a two-stream instability, corresponding to radial density modulations, in this geometry. PIC simulations confirm the semianalytical results because there is no evidence of instabilities. In contrast, we have shown that the system is unstable to azimuthal filamentation in the semi-analytical approach, which PIC simulations confirm. 


\section{ACKNOWLEDGMENTS}

The authors acknowledge funding from U.K. EPSRC (EP/J018171/1, EP/N028694/1), EU H2020 Research and Innovation Programme LASERLAB EUROPE (654148) and EuPRAXIA (653782). Data associated with research published in this paper is available at https://doi.org/10.15129/5251197a-7368-4d27-8f46-1eaedd27f418.

\section{REFERENCES}

[1] Hwang, K.-J., Goldstein, M. L., Kuznetsova, M. M., Wang, Y., Vias, A. F., and Sibeck, D. G., "The first in situ observation of kelvin-helmholtz waves at high-latitude magnetopause during strongly dawnward interplanetary magnetic field conditions," Journal of Geophysical Research: Space Physics 117(A8) (2012).

[2] Bret, A., Gremillet, L., and Dieckmann, M. E., "Multidimensional electron beam-plasma instabilities in the relativistic regime," Physics of Plasmas 17(12), 120501 (2010).

[3] Goldston, R. J. and Rutherford, P. H., [Introduction to plasma physics], Taylor and Francis Group (2005).

[4] Krall, N. A. and Trivelpiece, A. W., [Principles of plasma physics], San Francisco Pr. (1986).

[5] Weibel, E. S., "Spontaneously growing transverse waves in a plasma due to an anisotropic velocity distribution," Phys. Rev. Lett. 2, 83-84 (Feb 1959).

[6] Fried, B. D., "Mechanism for instability of transverse plasma waves," The Physics of Fluids 2(3), 337-337 (1959).

[7] Bohm, D. and Gross, E. P., "Theory of plasma oscillations. a. origin of medium-like behavior," Phys. Rev. 75, 1851-1864 (Jun 1949).

[8] Tajima, T. and Dawson, J. M., "Laser electron accelerator," Phys. Rev. Lett. 43, 267-270 (Jul 1979).

[9] Bret, A., Firpo, M.-C., and Deutsch, C., "Bridging the gap between two stream and filamentation instabilities," Laser and Particle Beams 23(3), 375383 (2005).

[10] Bret, A., "Hierarchy of instabilities for two counter-streaming magnetized pair beams," Physics of Plasmas 23(6), 062122 (2016).

[11] Arber, T. D., Bennett, K., Brady, C. S., Lawrence-Douglas, A., Ramsay, M. G., Sircombe, N. J., Gillies, P., Evans, R. G., Schmitz, H., Bell, A. R., and Ridgers, C. P., "Contemporary particle-in-cell approach to laser-plasma modelling," Plasma Physics and Controlled Fusion 57, 1-26 (Nov. 2015). 